\title{
Serum Amino Acid Profiles in Normal Subjects and in Patients with or at Risk of Alzheimer Dementia
}

\author{
Gaetano Corso $^{a}$ Adriana Cristofano $^{b}$ Nadia Sapere $^{b}$ \\ Giancarlo la Marcac, d Antonella Angiolillo ${ }^{b}$ Michela Vitale ${ }^{b}$ \\ Roberto Fratangelo ${ }^{b}$ Teresa Lombardi ${ }^{b}$ Carola Porcile ${ }^{b}$ \\ Mariano Intrieri ${ }^{b}$ Alfonso Di Costanzo ${ }^{b}$

\begin{abstract}
a Department of Clinical and Experimental Medicine, University of Foggia, Foggia, Italy; ${ }^{b}$ Centre for Research and Training in Medicine for Aging, Department of Medicine and Health Sciences "Vincenzo Tiberio," University of Molise, Campobasso, Italy; 'Newborn Screening, Biochemistry and Pharmacology Laboratories, Clinic of Pediatric Neurology, Meyer Children's Hospital, Florence, Italy; ${ }^{\mathrm{d} D e p a r t m e n t}$ of Neurosciences, Psychology, Pharmacology, and Child Health, University of Florence, Florence, Italy
\end{abstract}

\section{Keywords}

Alzheimer disease - Mild cognitive impairment - Subjective memory complaint - Amino acids . Tandem mass spectrometry

\section{Abstract}

Background/Aims: Abnormalities in the plasma amino acid profile have been reported in Alzheimer disease (AD), but no data exist for the prodromal phase characterized by subjective memory complaint (SMC). It was our aim to understand if serum amino acid levels change along the continuum from normal to $A D$, and to identify possible diagnostic biomarkers. Methods: Serum levels of 15 amino acids and 2 organic acids were determined in 4 groups of participants - 29 with probable AD, 18 with mild cognitive impairment (MCI), 24 with SMC, and 46 cognitively healthy subjects (HS) - by electrospray tandem mass spectrometry. $\boldsymbol{R e}$ sults: Glutamate, aspartate, and phenylalanine progressively decreased, while citrulline, argininosuccinate, and homocitrulline progressively increased, from HS over SMC and MCI to AD. The panel including these 6 amino acids and 4 ratios (glutamate/citrulline, citrulline/phenylalanine, leucine plus isoleucine/phenylalanine, and arginine/phenylalanine) discriminated AD from HS with about $96 \%$ accuracy. Other panels including 20 biomarkers discriminated SMC or MCI from AD or HS with an accuracy ranging from 88 to $75 \%$. Conclusion: Amino acids contribute to a characteristic metabotype during the progression of $A D$ along the continuum from health to frank dementia, and their monitoring in elderly individuals might help to detect at-risk subjects. 


\section{Introduction}

Alzheimer disease (AD) is the most common neurodegenerative disorder and is characterized by a progressive loss of memory and executive function. In 2016, an estimated 5.2 million Americans aged 65 years or older are living with AD, and every $66 \mathrm{~s}$, a new case of $\mathrm{AD}$ is diagnosed [1]. The evidence of brain atrophy and cerebrospinal fluid (CSF) abnormalities in cognitively normal subjects suggests that the neuropathological process might precede clinical symptoms by several years [2-5], and this proposes the existence of a cognitive continuum that moves from a normal cognitive state to full-blown dementia. Currently, 2 clinical conditions, known as mild cognitive impairment (MCI) and subjective memory complaint (SMC), have been identified as the earliest stages of disease and are both associated with an increased risk for $\mathrm{AD}[1,6,7]$. $\mathrm{MCI}$ is defined as impairment of 1 or more cognitive domains that is worse than expected for the age and level of education of an individual, but is not severe enough to significantly compromise daily activities [6]. SMC indicates a self-reported memory decline, which may or may not be perceived by others, in the absence of pathological results on neuropsychological tests [7].

To date, the molecular mechanisms associated with $\mathrm{AD}$ have remained elusive, and no drug can reverse the pathological process [1]. Thus, one of the major challenges of modern research is to identify highly reliable biomarkers for an early diagnosis of $\mathrm{AD}$, in the hope of defining a therapeutic window in which the neural substrate is still responsive to therapy $[8,9]$. Unfortunately, the biomarkers evaluated to date - including CSF levels of total tau, phosphorylated tau, and amyloid- $\beta_{42}\left(A \beta_{42}\right)$ - have not shown the accuracy and reliability needed to diagnose $\mathrm{AD}$ [10]. In the last years, metabolomics has been recognized as a promising approach to adequately defining the at-risk state of AD. There is, in fact, a growing body of evidence that $\mathrm{AD}$ and its earlier stages may have a metabolic biosignature easily inferable from peripheral blood by minimally invasive and relatively inexpensive procedures $[8,11]$.

Amino acids not only are indispensable for humans as a nutrient but also play essential roles in the control and regulation of crucial functions of the human body [12,13]. In the brain, free amino acids are important for neurotransmission and receptor signaling pathways, and they are involved in neurotoxicity. Therefore, biochemical pathways involving amino acids might be altered in $\mathrm{AD}$, and changes in free amino acid levels might be an early indicator of neurodegeneration $[14,15]$. However, the literature on amino acid analysis in AD provides contradictory results. For example, some researchers have found no significant differences in CSF or blood levels of glutamate (Glu) between normal and AD patients [16,17], whereas others have shown that this amino acid was elevated $[18,19]$ or reduced $[20,21]$ in AD. Similarly, aspartate (Asp) levels in CSF or blood have been found to be increased [19,21], reduced [20], or unchanged [16] in AD compared to normal subjects. For these reasons, further studies are needed to clarify the role of amino acid alterations in the pathogenesis of $\mathrm{AD}$ and to gain new insights into early detection and prevention of this devastating disease.

In the present study, we determined the serum levels of 15 amino acids and 2 organic acids in different groups of subjects along the continuum from normal over SMC and MCI to AD with a double aim: (a) to understand if homeostasis of amino acids is implicated in the onset and progression of $\mathrm{AD}$ and (b) to identify possible diagnostic biomarkers. To the best of our knowledge, this research is the first to investigate serum levels of amino acids in subjects with SMC. 
Corso et al.: Serum Amino Acid Profiles in Normal Subjects and in Patients with or at Risk of Alzheimer Dementia

Table 1. Demographic and clinical characteristics of the study groups

\begin{tabular}{|c|c|c|c|c|}
\hline & \multicolumn{4}{|l|}{ Group } \\
\hline & $\mathrm{AD}(n=29)$ & $\operatorname{MCI}(n=18)$ & $\operatorname{SMC}(n=24)$ & HS $(n=46)$ \\
\hline Mean age $\pm S D$, years & $71.4 \pm 9.5$ & $69.4 \pm 8.2$ & $66.0 \pm 7.6$ & $65.0 \pm 7.1$ \\
\hline \multicolumn{5}{|l|}{ Gender, $n(\%)$} \\
\hline Male & $7(5.9)$ & $4(3.4)$ & $8(6.8)$ & $20(17.1)$ \\
\hline Female & $22(18.8)$ & $14(11.97)$ & $16(13.7)$ & $26(22.2)$ \\
\hline Mean educational level \pm SD, years & $8.9 \pm 4.9$ & $9.3 \pm 4.14$ & $11.8 \pm 3.0$ & $12.4 \pm 3.3$ \\
\hline Mean BMI \pm SD & $25.8 \pm 5.4$ & $27.78 \pm 3.6$ & $27.52 \pm 4.1$ & $28.56 \pm 4.1$ \\
\hline Mean MMSE score \pm SD & $12.6 \pm 8.0$ & $26.7 \pm 2.2$ & $29.6 \pm 0.7$ & $29.6+0.7$ \\
\hline \multicolumn{5}{|l|}{ Medical history, $n(\%)$} \\
\hline Smoking $^{\mathrm{a}}$ & $8(27.6)$ & $5(27.7)$ & $5(20.8)$ & $12(26.1)$ \\
\hline Dyslipidemia & $10(34.5)$ & $5(27.8)$ & $6(25.0)$ & $12(26.1)$ \\
\hline Diabetes & $7(24.1)$ & $4(22.2)$ & $4(16.7)$ & $6(13.0)$ \\
\hline Hypertension & $14(48.3)$ & $9(50.0)$ & $10(41.7)$ & $18(39.1)$ \\
\hline Arrhythmia & $2(6.9)$ & $2(11.1)$ & $2(8.3)$ & $3(6.5)$ \\
\hline Myocardial infarction & $3(10.3)$ & $1(5.5)$ & $2(8.3)$ & $2(4.3)$ \\
\hline Heart failure ${ }^{b}$ & $2(6.9)$ & - & - & $2(4.3)$ \\
\hline TIA/stroke & $2(6.9)$ & $1(5.5)$ & $2(8.3)$ & $1(2.2)$ \\
\hline Chronic kidney disease $^{c}$ & $1(3.4)$ & - & - & - \\
\hline Prior tumors & $3(10.3)$ & $1(5.5)$ & $3(12.5)$ & $5(10.9)$ \\
\hline \multicolumn{5}{|l|}{ Drugs, $n(\%)$} \\
\hline Antihypertensive & $14(48.3)$ & $9(50.0)$ & $10(41.7)$ & $18(39.1)$ \\
\hline Lipid-lowering & $5(17.2)$ & $5(27.8)$ & $6(25.0)$ & $9(19.6)$ \\
\hline Hypoglycemic & $7(24.1)$ & $4(22.2)$ & $4(16.7)$ & $6(13.0)$ \\
\hline Antiacid & $8(27.6)$ & $3(16.7)$ & $6(25.0)$ & $8(17.4)$ \\
\hline Antiplatelet & $7(24.1)$ & $2(11.1)$ & $5(20.8)$ & $7(12.2)$ \\
\hline Supplements & $5(17.2)$ & $3(16.7)$ & $5(20.8)$ & $6(13.0)$ \\
\hline
\end{tabular}

-, none; $\mathrm{AD}$, Alzheimer disease; MCI, mild cognitive impairment; SMC, subjective memory complaint; HS, healthy subjects; BMI, body mass index; MMSE, Mini-Mental State Examination; TIA, transient ischemic attack. ${ }^{\text {a }}$ Current or former smoker. ${ }^{\mathrm{b}}$ Subjects in NYHA (New York Heart Association) class I-II. ${ }^{\mathrm{c}}$ Subjects with a glomerular filtration rate $>30 \mathrm{~mL} / \mathrm{min} / 1.73 \mathrm{~m}^{2}$.

\section{Subjects and Methods}

\section{Subjects}

A total of 117 participants were consecutively recruited from the Centre for Research and Training in Medicine for Aging (CeRMA), University of Molise. They were divided into 4 groups: 29 participants with probable AD (7 males, 22 females; mean age \pm SD: $71.4 \pm 9.5$ years), 18 with amnestic MCI (4 males, 14 females; $69.4 \pm 8.2$ years), 24 with SMC (8 males, 16 females; $66.0 \pm 7.6$ years), and 46 cognitively healthy subjects (HS; 20 males, 26 females; $65.0 \pm 7.1$ years). Table 1 reports the clinical and demographic characteristics of the 4 groups of participants. The body mass index (BMI) of the AD patients was slightly lower than that of the other groups (Table 1$)$, but the differences were not significant $(F=2.250 ; \mathrm{df}=3,113$; $p=0.086$ ). The Mini-Nutritional Assessment (MNA) showed that all participants were well nourished (MNA score $>23.5$ ), with the exception of 11 AD patients, who were at risk of malnutrition (MNA score between 18 and 23) but not malnourished.

Patients with probable AD were diagnosed according to National Institute on AgingAlzheimer's Association (NIA-AA) criteria [22] and fulfilled the criteria for the "probable AD with documented decline" category. They presented with Mini-Mental State Examination (MMSE) scores $<24$ [23] and Clinical Dementia Rating (CDR) scores $>0.5$ [24]. Subjects with amnestic MCI 
met the NIA-AA diagnostic criteria for MCI due to AD [25], had MMSE scores $>24$ and CDR scores of 0.5 , and showed memory impairment as assessed via age/sex/education-adjusted scores on at least 1 of the following tests: (1) Rey's word list, immediate and delayed recall [26], and (2) prose memory, immediate and delayed [27]. Participants with SMC stated that their memory function had deteriorated compared to earlier stages in life, reported that the time of onset was in adulthood, had a score of $\geq 25$ on the Memory Complaint Questionnaire (MAC-Q) [28] and showed normal objective memory performance on the Rey and prose memory tests [7].

To summarize, MCI subjects showed both subjective and objective memory impairment, SMC participants presented only memory complaints with a normal score on the memory tests, and HS showed neither subjective nor objective memory impairment. To rule out other potential causes of cognitive impairment, all participants underwent blood tests (including a full blood count and assessments of the erythrocyte sedimentation rate, urea and electrolytes, thyroid function, vitamin $\mathrm{B}_{12}$, and folate); furthermore, all patients with $\mathrm{AD}$ and $\mathrm{MCI}$, and 20 of the 24 subjects with SMC, underwent brain imaging. Depression was also ruled out using the Geriatric Depression Scale-Short Form (GDS-SF) [29]: participants with a GDS-SF score of $\geq 6$ were excluded from the study. The patients on treatment with cerebroactive drugs underwent a washout period of at least 14 days before assessment.

This study was conducted in accordance with the ethical principles stated in the Declaration of Helsinki, as well as with approved national and international guidelines for human research. The Ethics Committee of the University of Molise reviewed and approved this study, and written informed consent was required from the participants or caregivers.

\section{Blood Collection}

Blood collection was done between 8:00 and 8:30 a.m. after an overnight fasting of at least $12 \mathrm{~h}$. Venous blood was collected into Vacutainer serum tubes (Becton \& Dickinson, Milan, Italy) and centrifuged within $4 \mathrm{~h}$. All serum samples were stored at $-80^{\circ} \mathrm{C}$ until the preparation of spots and shipment to the analytical laboratory. To prepare spot serum from patients, $20 \mu \mathrm{L}$ of each serum were spotted on filter paper (Whatman 903; Whatman GmbH, Dassel, Germany), dried, and sent by courier to the laboratory at room temperature. The technicians of the analytical laboratory were blinded to the sample identification codes.

\section{Metabolite Analysis}

Targeted analysis of amino acids and organic acids was performed by tandem mass spectrometry (MS/MS) according to the conventional validated method as previously reported $[30,31]$. Briefly, a dried serum spot was punched into a microplate (96-well) and $200 \mu \mathrm{L}$ of methanol containing labeled amino acid standards were added. The labeled internal standards of amino acids were purchased from Cambridge Isotope Laboratories (Andover, MA, USA); a stock solution was made in methanol. The standard concentrations for the amino acids were in the range of 7.6-152 $\mu \mathrm{mol} / \mathrm{L}$; the daily working solution was made by dilution of the stock solution (1:200) using methanol:water 90:10 v/v.

The spots were shaken on a vortex system for $20 \mathrm{~min}$. After that, the solution containing the extracted amino acids was transferred to a new microplate and dried under a nitrogen flow at $50^{\circ} \mathrm{C}$. The dried amino acids were derivatized to butyl esters using $n$-butanol with $\mathrm{HCl}$ ( $3 \mathrm{~mol} / \mathrm{L}$ ) at $65^{\circ} \mathrm{C}$ for $25 \mathrm{~min}$. Then the sample was dried again and resuspended in $200 \mu \mathrm{L}$ of an aqueous solution of $50 \%$ acetonitrile containing $0.1 \%$ formic acid (mobile phase), after which $40 \mu \mathrm{L}$ were injected using flow injection analysis into a triple quadrupole mass spectrometer equipped with a TurboIonSpray source (API 4000; Applied Biosystems-MDS Sciex, Toronto, ON, Canada). The TurbolonSpray source was operated in positive ion mode with a needle potential of $+5,500 \mathrm{~V}$ and with a "Turbo" gas flow of $10 \mathrm{~L} / \mathrm{min}$ of air heated at $150^{\circ} \mathrm{C}$ (nominal heat gun temperature). 
Corso et al:: Serum Amino Acid Profiles in Normal Subjects and in Patients with or at Risk of Alzheimer Dementia

Mass calibration and resolution adjustments to the resolving quadrupoles were performed automatically using a PPG solution $\left(10^{-4} \mathrm{~mol} / \mathrm{L}\right)$ introduced via an infusion pump. The peak width was set on both resolving quadrupoles at 0.7 Th (measured at half height of peak) for all MS and MS/MS experiments. Collisional activated dissociation MS/MS was performed in the LINAC Q2 collision cell, operating with 10 mTorr pressure of nitrogen as a collision gas. The declustering potential and collision energy were automatically optimized for amino acids using the Analyst 1.4 software; the resulting values of the declustering potential and collision energy were $20 \mathrm{~V}$ and $32 \mathrm{eV}$, respectively.

Concentrations of the amino acids were calculated automatically using the ChemoView software (API 4000; AB SCIEX). The targeted analysis of the metabolic profiles was focused on the estimation of alanine (Ala), arginine (Arg), argininosuccinic acid (ASA), Asp, citrulline (Cit), formiminoglutamic acid, Glu, glycine (Gly), homocitrulline (Homo-Cit), hawkinsin, methionine (Met), phenylalanine (Phe), ornithine (Orn), succinylacetone (SUAC), tyrosine (Tyr), valine (Val), leucine plus isoleucine (Xle). In addition, 18 amino acid molar ratios (Phe/ Tyr, Val/Phe, Xle/Phe, Xle/Ala, Xle/Tyr, Met/Phe, Met/Tyr, Met/Xle, Met/Cit, Glu/Cit, Ala/Cit, Orn/Cit, Cit/Phe, Arg/Phe, Arg/Ala, Arg/Orn, Cit/Arg, and ASA/Arg) were calculated and used as additional markers. The results of the quality control of amino acid and organic acid measurements are reported in online supplementary Table 1 (for all online suppl. material, see www.karger.com/doi/10.1159/000466688). The intra-assay variability (CV\%), obtained analyzing 8 quality controls with different levels of concentration, was $<20 \%$ (ranging from 3.5 to $19.9 \%$ ) (online suppl. Table 1 ).

\section{Statistical Analysis and Data Processing}

The data were analyzed using the SPSS statistical software package (version 17.0; SPSS Inc., Chicago, IL, USA). Variables were examined for outliers and extreme values by means of box and normal quantile-quantile plots, as well as with Shapiro-Wilk and KolmogorovSmirnov tests. When normal distribution could not be accepted, variable transformations (square, square root, logarithmic, reciprocal of square root, or reciprocal transformations) were reviewed; the reciprocal of square root of ASA levels, the square root of Met/Cit, Orn/ Cit, Cit/Phe, Arg/Phe, Arg/Ala, Arg/Orn, and Cit/Arg ratios, and, finally, the reciprocal of Gly, Homo-Cit, and SUAC concentrations helped to improve the distribution shape.

One-way multi- and univariate analyses of variance (ANOVAs) were used to evaluated group differences (HS vs. SMC vs. MCI vs. AD). Age, gender, educational level, and BMI were included into the model as covariates. The assumption of equality of variance was assessed by means of Levene's test. Finally, post hoc pairwise multiple comparison using Bonferroni's correction was performed in order to detect significant differences $(p<0.05)$ between specific means. The degree of linear association between MMSE score or educational level and amino acid concentrations in each group was evaluated by the Spearman rank correlation coefficient, using Bonferroni's correction for multiple comparisons. The $\chi^{2}$ test was used to assess differences in comorbidity and drug intake between groups.

Multivariate statistical analysis was also performed, using projection methods as implemented in the MetaboAnalyst 3.0 online package [32]. Principal component analysis (PCA) was first applied to detect sample metabolite trends and clustering in an unsupervised manner, and the partial least-squares discriminant analysis (PLS-DA) was applied to reinforce classification and to better identify clustering. The concentrations of all variables analyzed were preprocessed by normalization by the sum, transformed by log normalization, and with mean-centered and auto scaling before building the model. The model quality was evaluated by the goodness-of-fit parameter $\left(R^{2}\right)$ and the goodness-of-prediction parameter $\left(Q^{2}\right)$. 
Corso et al.: Serum Amino Acid Profiles in Normal Subjects and in Patients with or at Risk of Alzheimer Dementia

Table 2. Amino acid levels $(\mu \mathrm{mol} / \mathrm{L})$ in serum of patients and healthy subjects

\begin{tabular}{|c|c|c|c|c|c|c|c|c|c|c|c|c|}
\hline \multirow{3}{*}{$\begin{array}{l}\text { Amino } \\
\text { acids }\end{array}$} & \multirow[t]{3}{*}{$\mathrm{AD}(n=29)$} & \multirow[t]{3}{*}{$\operatorname{MCI}(n=18)$} & \multirow[t]{3}{*}{$\operatorname{SMC}(n=24)$} & \multirow[t]{3}{*}{ HS $(n=46)$} & \multirow{3}{*}{$\begin{array}{l}\text { ANOVA }^{\text {a }} \\
F(3,109)\end{array}$} & \multicolumn{7}{|c|}{ Pairwise multiple comparisons ${ }^{\mathrm{b}}$} \\
\hline & & & & & & \multirow[t]{2}{*}{$p$} & $\mathrm{AD}$ & $\mathrm{AD}$ & $\mathrm{AD}$ & MCI & HS & HS \\
\hline & & & & & & & $\begin{array}{l}\text { vs. } \\
\text { MCI }\end{array}$ & $\begin{array}{l}\text { vs. } \\
\text { SMC }\end{array}$ & $\begin{array}{l}\text { vs. } \\
\text { HS }\end{array}$ & $\begin{array}{l}\text { vs. } \\
\text { SMC }\end{array}$ & $\begin{array}{l}\text { vs. } \\
\text { MCI }\end{array}$ & $\begin{array}{l}\text { vS. } \\
\text { SMC }\end{array}$ \\
\hline Ala & $195.58 \pm 40.93$ & $200.96 \pm 29.59$ & $195.9 \pm 40.18$ & $216.51 \pm 42.75$ & 2.710 & 0.049 & ns & ns & ns & ns & ns & ns \\
\hline Arg & $102.83 \pm 18.79$ & $91.13 \pm 18.16$ & $98.37 \pm 18.55$ & $96.75 \pm 22.92$ & 1.108 & 0.349 & NA & NA & NA & NA & NA & NA \\
\hline ASA & $0.24 \pm 0.09$ & $0.19 \pm 0.04$ & $0.18 \pm 0.06$ & $0.17 \pm 0.05$ & 4.207 & 0.007 & ns & 0.002 & $<0.001$ & ns & ns & ns \\
\hline Asp & $44.76 \pm 8.11$ & $50.28 \pm 8.91$ & $54.04 \pm 7.54$ & $57.79 \pm 10.97$ & 9.911 & $<0.001$ & ns & 0.003 & $<0.001$ & ns & 0.028 & ns \\
\hline Cit & $48.69 \pm 14.64$ & $44.33 \pm 9.97$ & $43.62 \pm 8.81$ & $38.86 \pm 8.13$ & 3.247 & 0.025 & ns & ns & 0.001 & ns & ns & ns \\
\hline FIGLU & $0.16 \pm 0.04$ & $0.15 \pm 0.03$ & $0.15 \pm 0.04$ & $0.15 \pm 0.03$ & 1.045 & 0.376 & NA & NA & NA & NA & NA & NA \\
\hline Glu & $101.70 \pm 15.33$ & $114.52 \pm 18.67$ & $121.15 \pm 21.46$ & $132.31 \pm 20.91$ & 10.273 & $<0.001$ & ns & 0.003 & $<0.001$ & ns & 0.008 & ns \\
\hline Gly & $497.06 \pm 76.33$ & $482.38 \pm 63.85$ & $502.24 \pm 93.76$ & $484.02 \pm 64.33$ & 0.264 & 0.851 & NA & NA & NA & NA & NA & NA \\
\hline Homo-Cit & $1.46 \pm 0.62$ & $1.35 \pm 0.35$ & $1.21 \pm 0.36$ & $1.06 \pm 0.20$ & 3.160 & 0.028 & ns & ns & 0.001 & ns & 0.008 & ns \\
\hline Hawk & $0.10 \pm 0.03$ & $0.09 \pm 0.02$ & $0.08 \pm 0.02$ & $0.10 \pm 0.03$ & 5.314 & 0.002 & ns & 0.015 & ns & ns & ns & 0.003 \\
\hline Met & $28.57 \pm 5.71$ & $27.53 \pm 6.79$ & $27.07 \pm 4.15$ & $31.91 \pm 6.10$ & 4.767 & 0.004 & ns & ns & ns & ns & 0.044 & 0.007 \\
\hline Orn & $33.08 \pm 4.27$ & $33.56 \pm 4.28$ & $33.19 \pm 4.02$ & $34.39 \pm 4.56$ & 0.656 & 0.581 & NA & NA & NA & NA & NA & NA \\
\hline Phe & $81.68 \pm 8.84$ & $87.47 \pm 8.01$ & $90.44 \pm 8.05$ & $95.86 \pm 11.45$ & 10.758 & $<0.001$ & ns & 0.009 & $<0.001$ & ns & 0.014 & ns \\
\hline SUAC & $1.37 \pm 0.11$ & $1.49 \pm 0.14$ & $1.42 \pm 0.09$ & $1.55 \pm 0.14$ & 11.709 & $<0.001$ & 0.006 & ns & $<0.001$ & ns & ns & 0.001 \\
\hline Tyr & $91.25 \pm 17.01$ & $95.98 \pm 14.38$ & $93.21 \pm 11.10$ & $103.30 \pm 18.30$ & 3.185 & 0.027 & ns & ns & 0.013 & ns & ns & ns \\
\hline Val & $174.46 \pm 19.75$ & $193.04 \pm 26.80$ & $207.08 \pm 36.24$ & $197.05 \pm 25.25$ & 5.501 & 0.001 & ns & $<0.001$ & 0.004 & ns & ns & ns \\
\hline Xle & $148.55 \pm 21.42$ & $148.61 \pm 16.46$ & $146.86 \pm 21.05$ & $159.85 \pm 22.67$ & 2.233 & 0.088 & NA & NA & NA & NA & NA & NA \\
\hline
\end{tabular}

Results are reported as the mean + SD. Statistical analysis was performed by ANOVA. NA, not applicable; ns, not significant; AD, Alzheimer disease; MCI, mild cognitive impairment; SMC, subjective memory complaint; HS, healthy subjects; FIGLU, formiminoglutamic acid; Hawk, hawkinsin. For the explanations of the remaining abbreviations, see Subjects and Methods. ${ }^{a}$ Covariates (age, gender, educational level, and body mass index). ${ }^{b}$ Bonferroni post hoc analysis.

Table 3. Amino acid molar ratios in serum of patients and healthy subjects

\begin{tabular}{|c|c|c|c|c|c|c|c|c|c|c|c|c|}
\hline \multirow[t]{2}{*}{ Ratios } & \multirow[t]{2}{*}{$\mathrm{AD}(n=29)$} & \multirow[t]{2}{*}{$\operatorname{MCI}(n=18)$} & \multirow[t]{2}{*}{$\operatorname{SMC}(n=24)$} & \multirow[t]{2}{*}{ HS $(n=46)$} & \multirow{2}{*}{$\begin{array}{l}\text { ANOVA }^{\mathrm{a}} \\
F(3,109)\end{array}$} & \multicolumn{7}{|c|}{ Pairwise multiple comparisons $\mathrm{s}^{\mathrm{b}}$} \\
\hline & & & & & & $p$ & $\begin{array}{l}\mathrm{AD} \\
\text { vs. } \\
\mathrm{MCI}\end{array}$ & $\begin{array}{l}\text { AD } \\
\text { vs. } \\
\text { SMC }\end{array}$ & $\begin{array}{l}\text { AD } \\
\text { vs. } \\
\text { HS }\end{array}$ & $\begin{array}{l}\text { MCI } \\
\text { vs. } \\
\text { SMC }\end{array}$ & $\begin{array}{l}\text { HS } \\
\text { vs. } \\
\text { MCI }\end{array}$ & $\begin{array}{l}\text { HS } \\
\text { vs. } \\
\text { SMC }\end{array}$ \\
\hline $\mathrm{Ala} / \mathrm{Cit}$ & $4.35 \pm 1.61$ & $4.58 \pm 0.81$ & $4.64 \pm 1.32$ & $5.77 \pm 1.53$ & 6.478 & $<0.001$ & ns & ns & $<0.001$ & ns & 0.018 & 0.012 \\
\hline Arg/Ala & $0.55 \pm 0.15$ & $0.46 \pm 0.11$ & $0.53 \pm 0.16$ & $0.45 \pm 0.11$ & 3.888 & 0.011 & ns & ns & 0.020 & ns & ns & ns \\
\hline Arg/Orn & $3.16 \pm 0.72$ & $2.75 \pm 0.61$ & $3.02 \pm 0.75$ & $2.81 \pm 0.51$ & 2.079 & 0.107 & NA & NA & NA & NA & NA & NA \\
\hline $\mathrm{Arg} / \mathrm{Phe}$ & $1.27 \pm 0.26$ & $1.04 \pm 0.20$ & $1.09 \pm 0.19$ & $1.01 \pm 0.18$ & 8.408 & $<0.001$ & 0.002 & 0.020 & $<0.001$ & ns & ns & ns \\
\hline ASA/Arg & $0.002 \pm 0.001$ & $0.002 \pm 0.0005$ & $0.002 \pm 0.0005$ & $0.002 \pm 0.0006$ & 1.945 & 0.127 & NA & NA & NA & NA & NA & NA \\
\hline Cit/Arg & $0.49 \pm 0.17$ & $0.50 \pm 0.12$ & $0.46 \pm 0.11$ & $0.41 \pm 0.09$ & 1.971 & 0.123 & NA & NA & NA & NA & NA & NA \\
\hline Cit/Phe & $0.60 \pm 0.18$ & $0.51 \pm 0.13$ & $0.61 \pm 0.58$ & $0.41 \pm 0.08$ & 5.206 & 0.002 & ns & ns & $<0.001$ & ns & ns & 0.007 \\
\hline Glu/Cit & $2.24 \pm 0.66$ & $2.64 \pm 0.64$ & $2.89 \pm 0.87$ & $3.52 \pm 0.79$ & 11.985 & $<0.001$ & ns & 0.014 & $<0.001$ & ns & $<0.001$ & 0.008 \\
\hline Met/Cit & $0.64 \pm 0.22$ & $0.64 \pm 0.15$ & $0.65 \pm 0.17$ & $0.84 \pm 0.17$ & 10.126 & $<0.001$ & ns & ns & $<0.001$ & ns & $<0.001$ & $<0.001$ \\
\hline Met/Phe & $0.35 \pm 0.06$ & $0.31 \pm 0.07$ & $0.30 \pm 0.04$ & $0.33 \pm 0.04$ & 3.999 & 0.010 & ns & 0.004 & ns & ns & ns & ns \\
\hline Met/Tyr & $0.32 \pm 0.05$ & $0.29 \pm 0.05$ & $0.30 \pm 0.04$ & $0.31 \pm 0.04$ & 2.339 & 0.077 & NA & NA & NA & NA & NA & NA \\
\hline Met/Xle & $0.19 \pm 0.03$ & $0.18 \pm 0.03$ & $0.19 \pm 0.02$ & $0.20 \pm 0.03$ & 2.153 & 0.098 & NA & NA & NA & NA & NA & NA \\
\hline Orn/Cit & $0.73 \pm 0.20$ & $0.78 \pm 0.19$ & $0.78 \pm 0.17$ & $0.91 \pm 0.19$ & 5.217 & 0.002 & ns & $\mathrm{ns}$ & $<0.001$ & ns & ns & 0.037 \\
\hline Phe/Tyr & $0.92 \pm 0.15$ & $0.93 \pm 0.14$ & $0.98 \pm 0.10$ & $0.94 \pm 0.12$ & 0.859 & 0.465 & NA & NA & NA & NA & NA & NA \\
\hline Val/Phe & $2.14 \pm 0.16$ & $2.20 \pm 0.29$ & $2.31 \pm 0.49$ & $2.05 \pm 0.23$ & 4.279 & 0.007 & ns & ns & ns & ns & ns & 0.005 \\
\hline Xle/Ala & $0.79 \pm 0.17$ & $0.75 \pm 0.10$ & $0.77 \pm 0.12$ & $0.75 \pm 0.11$ & 1.228 & 0.303 & NA & NA & NA & NA & NA & NA \\
\hline Xle/Phe & $1.82 \pm 0.16$ & $1.69 \pm 0.19$ & $1.62 \pm 0.15$ & $1.67 \pm 0.14$ & 8.226 & $<0.001$ & 0.049 & $<0.001$ & 0.001 & ns & ns & ns \\
\hline Xle/Tyr & $1.66 \pm 0.28$ & $1.57 \pm 0.20$ & $1.59 \pm 0.23$ & $1.57 \pm 0.17$ & 1.304 & 0.277 & NA & NA & NA & NA & NA & NA \\
\hline
\end{tabular}

Results are reported as the mean + SD. Statistical analysis was performed by ANOVA. NA, not applicable; ns, not significant; AD, Alzheimer disease; MCI, mild cognitive impairment; SMC, subjective memory complaint; HS, healthy subjects. For the explanations of the remaining abbreviations, see Subjects and Methods. ${ }^{\mathrm{a}}$ Covariates (age, gender, educational level, and body mass index). ${ }^{\mathrm{b}}$ Bonferroni post hoc analysis.

Pearson rank order was used to measure correlations between metabolites and molar ratios. The heat map was obtained using all the values of the best metabolites and molar ratios selected by the random forest algorithm. Classic and multivariate receiver operating characteristic (ROC) curve analyses were performed using the tool for biomarker analysis of the MetaboAnalyst 3.0 package [33]. This tool allows the evaluation of diagnostic power for each 


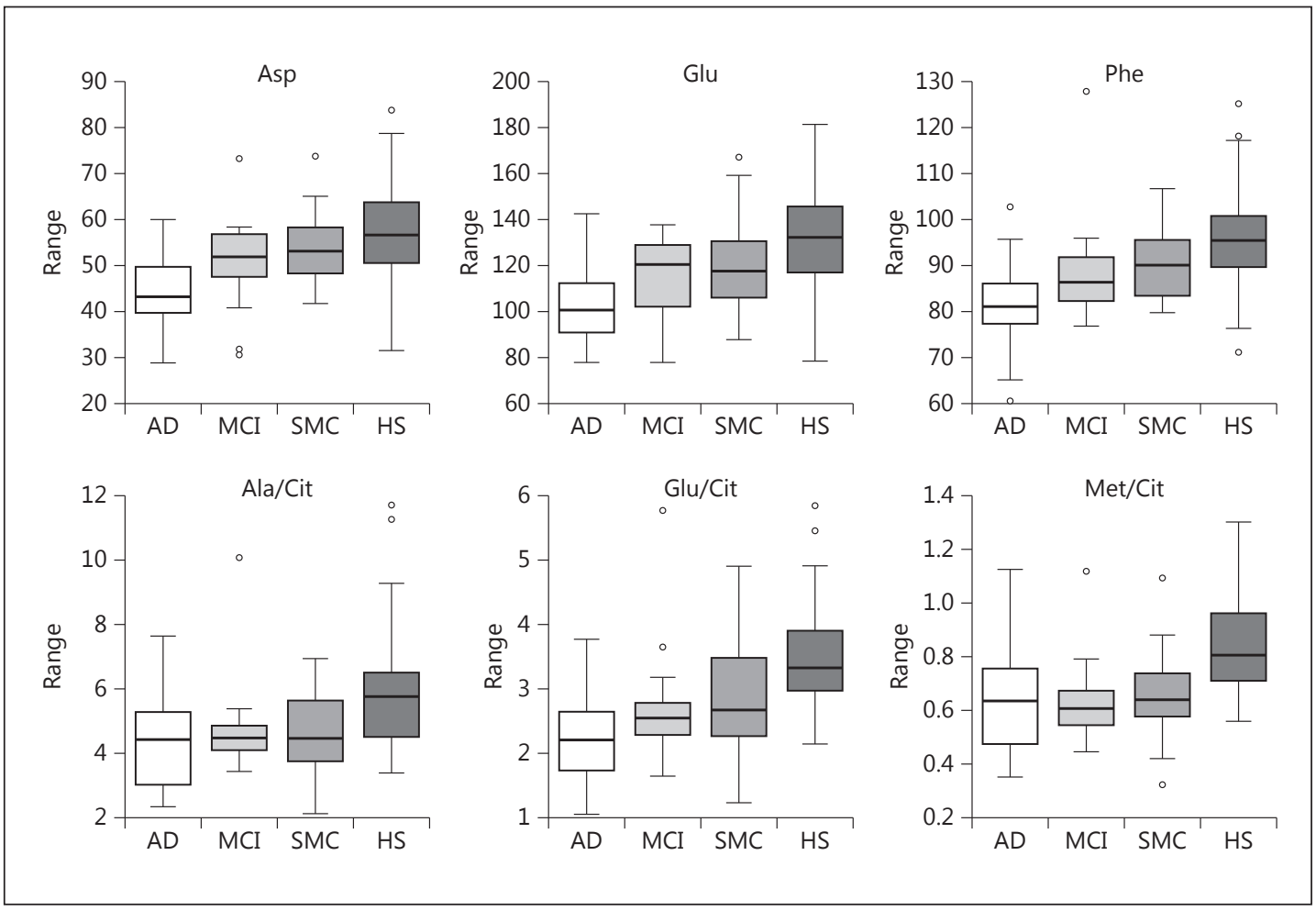

Fig. 1. Amino acids and molar ratios with a significant decreasing trend from HS to AD. The boxplots show the median (horizontal line in the box), 25th and 75th percentiles (edges of the box), maximum and minimum values (whiskers), and outliers $\left(^{\circ}\right.$ ) of Glu, Asp, and Phe serum concentrations ( $\mu \mathrm{mol} / \mathrm{L}$ ) and 3 molar ratios in the 4 groups of subjects. AD, Alzheimer disease; MCI, mild cognitive impairment; SMC, subjective memory complaint; HS, healthy subjects; Glu, glutamate, Asp, aspartate; Phe, phenylalanine; Ala, alanine; Met, methionine; Cit, citrulline.

amino acid and ratio calculating the area under the curve (AUC) and the confidence interval (CI). According to the criteria of Jones and Athanasiou [34], AUC of $>0.97,0.93-0.96,0.75-$ 0.92, and 0.6-0.74 were interpreted as "excellent," "very good," "good," and "reasonable," respectively. Before the ROC curve analyses, the data set was log transformed and scaled using the unit variance scaling method (mean centered and divided by the SD of each variable). Multivariate ROC curve analysis allows the identification of multiple biomarkers and assesses the classification performance of the generated models. Here, we used a well-established algorithm (i.e., the random forest algorithm) to select and cross-validate the most important features. In brief, the model was validated through repeated random subsampling cross-validation where, in each cross-validation, two-thirds of the samples are used to evaluate the importance of each feature. The generated model was then further validated by 1,000 permutation tests using the AUC as the performing measure.

\section{Results}

Multivariate ANOVA, including age, gender, educational level, and BMI as covariates, showed a statistically significant difference $(F=2.577 ; \mathrm{df}=108,228 ; p<0.001$; Pillai's trace $=1.649$; partial $\eta^{2}=0.550$ ) between groups (HS vs. SMC vs. MCI vs. AD). 
The results of the univariate ANOVAs and post hoc multiple comparisons are reported in Table 2. The mean concentrations of Glu $\left(F=10.273 ; \mathrm{df}=3,109 ; p<0.001\right.$; partial $\left.\eta^{2}=0.220\right)$, Asp $\left(F=9.911 ; \mathrm{df}=3,109 ; p<0.001 ;\right.$ partial $\left.\eta^{2}=0.214\right)$, and Phe $(F=10.758 ; \mathrm{df}=3,109 ; p<$ 0.001 ; partial $\eta^{2}=0.228$ ) showed a significant downward trend, decreasing from HS via SMC and MCI to AD (Table 2; Fig. 1). For all 3 metabolites (Glu, Asp, and Phe), pairwise multiple comparisons showed that the HS group was significantly different from the MCI $(p<0.05)$ and the $\mathrm{AD}(p<0.001)$ group, and the latter was significantly different from the SMC $(p<0.05)$ group. SUAC, Tyr, and Val also showed decreased serum concentrations in the AD group compared to HS (Table 2). On the other hand, a significant upward trend was detected for the serum levels of Cit $\left(F=3.247 ; \mathrm{df}=3,109 ; p=0.025 ;\right.$ partial $\left.\eta^{2}=0.082\right)$, ASA $(F=4.207 ; \mathrm{df}=3,109 ; p=0.007$; partial $\left.\eta^{2}=0.104\right)$, and Homo-Cit $\left(F=3.160 ; \mathrm{df}=3,109 ; p=0.028\right.$; partial $\left.\eta^{2}=0.080\right)$, progressively increasing from HS over SMC and MCI to AD (Table 2; Fig. 2). The comparison AD versus HS was always significant for these amino acids, while the comparison AD versus SMC was significant only for ASA and the comparison HS versus MCI only for Homo-Cit (Table 2).

The results of the univariate ANOVA and post hoc multiple comparisons for the molar ratios are reported in Table 3. Ten ratios, i.e., Ala/Cit, Arg/Ala, Arg/Phe, Cit/Phe, Glu/Cit, Met/ Cit, Met/Phe, Orn/Cit, Val/Phe, and Xle/Phe, changed significantly between the 4 groups. In particular, Ala/Cit, Arg/Phe, Glu/Cit, Met/Cit, and Xle/Phe showed at least 3 significant pairwise comparisons, including AD versus HS (Table 3). Ala/Cit, Glu/Cit, and Met/Cit decreased (Table 3; Fig. 1), while Arg/Phe, Cit/Phe, and Xle/Phe increased (Table 3; Fig. 2) from HS over SMC and $\mathrm{MCI}$ to AD. The Spearman rank correlation coefficients showed no significant correlation between MMSE or educational level and amino acid concentrations for each group. The $\chi^{2}$ test showed no significant differences in comorbidity or drug treatment between the groups.

Class PCA, used to check data homogeneity, detected neither subgroups nor strong outliers. Both PCA and PLS-DA showed that the first 2 components (PC1 and PC2) separated AD patients from HS well, while the MCI and SMC samples were found in the intermediate zone between AD and HS (Fig. 3a). The clusters of amino acids and molar ratios that strongly influenced the separation of HS from AD were Asp, Glu, Phe, Val, Met/Phe, Met/Tyr, Met/Xle, and Xle/Phe along the first principal component (PC1), and Ala, Arg, ASA, Cit, Homo-Cit, Ala/ Cit, Arg/Ala, Arg/Orn, Arg/Phe, Cit/Arg, Cit/Phe, Glu/Cit, Met/Cit, Orn/Cit, and Xle/Ala along the second principal component (PC2). Both amino acids and molar ratios differently contributed to the separation between groups, as represented by the loading plots of PC1 and PC2 (Fig. 3b). All loading values for the first 3 principal components are reported in online suppl. Table 2. The heat map obtained from multivariate analysis using the random forest algorithm confirmed the above-reported trends for some variations in amino acids and molar ratios between the 4 classes of subjects (Fig. 4). These findings were further confirmed by correlation analysis, showing that several amino acids, such as Ala, Arg, ASA, Asp, Cit, Gly, Glu, Met, Orn, Tyr, Val, and Xle, correlated well $(r> \pm 0.6)$ with each other as well as with several molar ratios (online suppl. Table 3).

The univariate ROC curve analysis was performed using all amino acids and their molar ratios as potential biomarkers. The first 10 biomarkers ranked by AUC and the related statistical significance for all pairwise comparisons are reported in online suppl. Table 4. The diagnostic accuracies of the selected biomarkers were as follows; good for AD versus HS (AUC from 0.90 to 0.75 ); good or reasonable for HS versus MCI (AUC from 0.86 to 0.70 ), AD versus SMC (AUC from 0.80 to 0.70 ), and HS versus SMC (AUC from 0.80 to 0.70 ); and reasonable for AD versus MCI (AUC from 0.75 to 0.67 ) and MCI versus SMC (AUC from 0.67 to 0.60 ).

Multivariate ROC curve analysis (Explorer) showed that the combination of the 10 biomarkers Glu, Glu/Cit, Cit/Phe, Asp, Xle/Phe, Arg/Phe, ASA, Homo-Cit, Phe, and Cit reached a very good power in discriminating AD from HS (AUC = 0.948; Fig. 5a). Anyway, good or very good diagnostic power was also obtained with the other models developed, using the first 3 


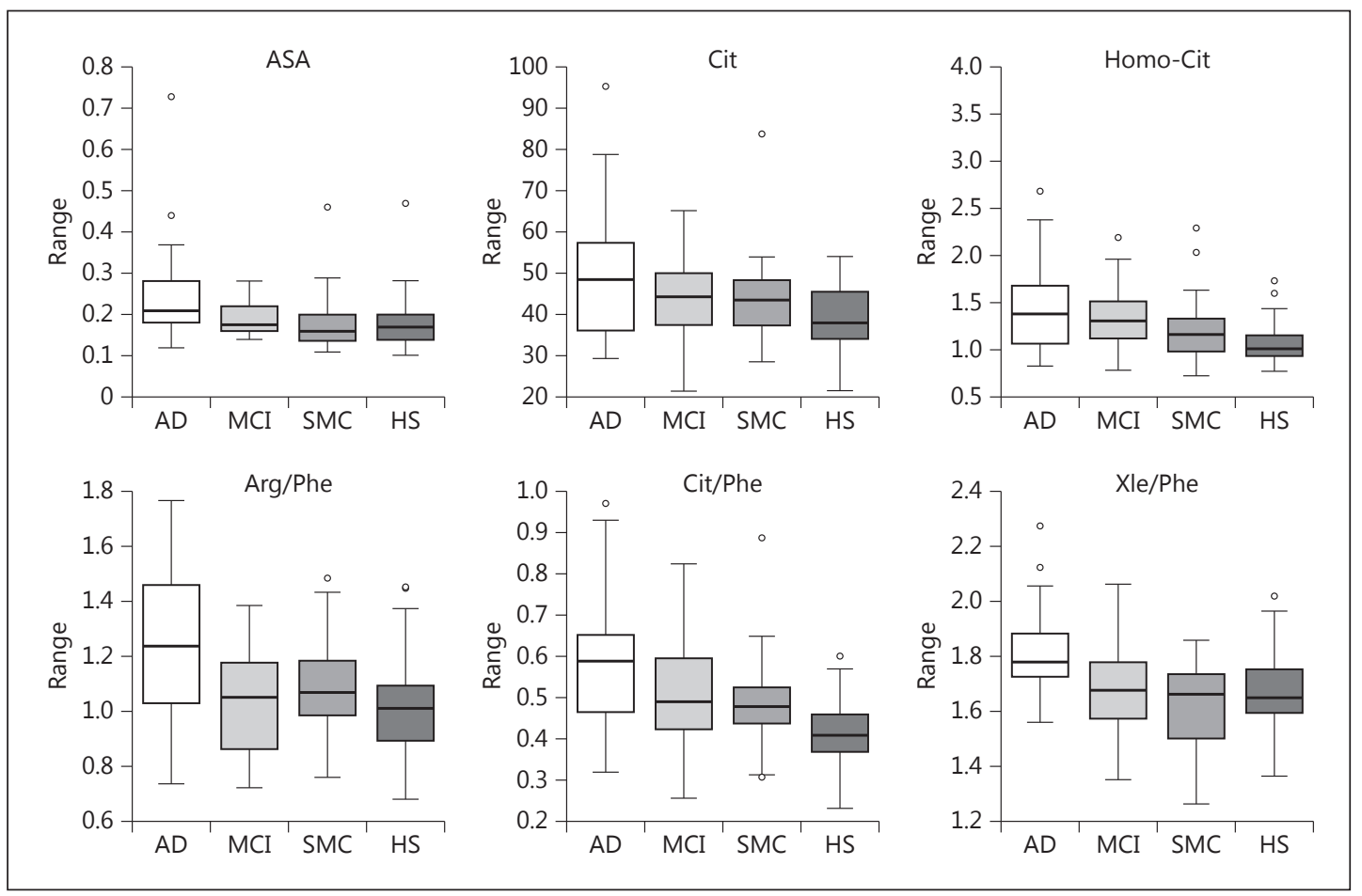

Fig. 2. Amino acids and molar ratios with a significant increasing trend from HS to AD. The boxplots (see Fig. 1 for details) of ASA, Cit, and Homo-Cit serum concentrations ( $\mu \mathrm{mol} / \mathrm{L}$ ) and 3 molar ratios in the 4 groups of subjects. AD, Alzheimer disease; MCI, mild cognitive impairment; SMC, subjective memory complaint; HS, healthy subjects; ASA, argininosuccinic acid; Cit, citrulline; Homo-Cit, homocitrulline; Arg, arginine; Phe, phenylalanine; Xle, leucine or isoleucine.

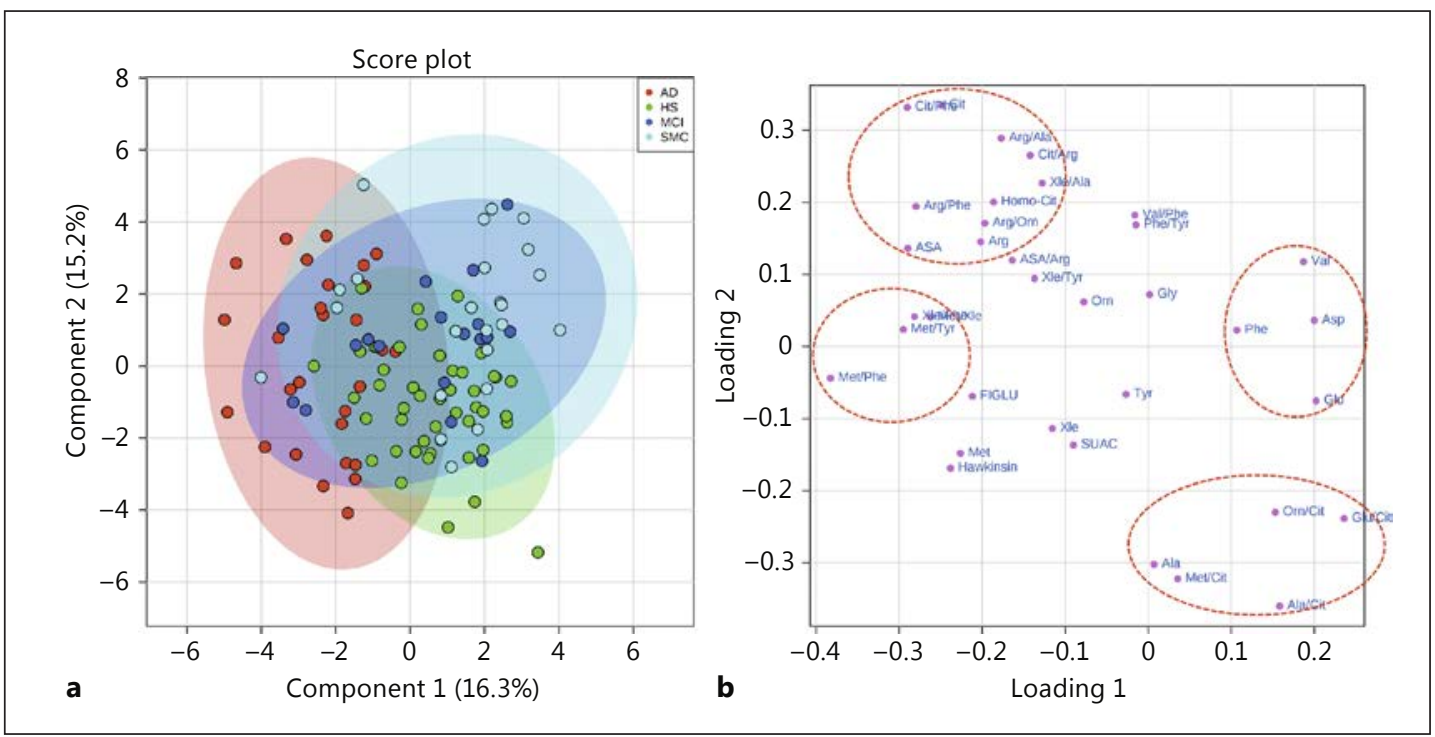

Fig. 3. Score and loading plots from partial least-squares discriminant analysis discriminating the 4 groups of subjects. Scores (a) and loadings (b) of the first 2 components explain the $31.5 \%$ of model variance. The AD samples (red) were well separated from HS (green), while MCI (blue) and SMC (light blue) clustered in an intermediate zone. AD, Alzheimer disease; MCI, mild cognitive impairment; SMC, subjective memory complaint; HS, healthy subjects; FIGLU, formiminoglutamic acid. For explanations of the remaining abbreviations, see Subjects and Methods. 


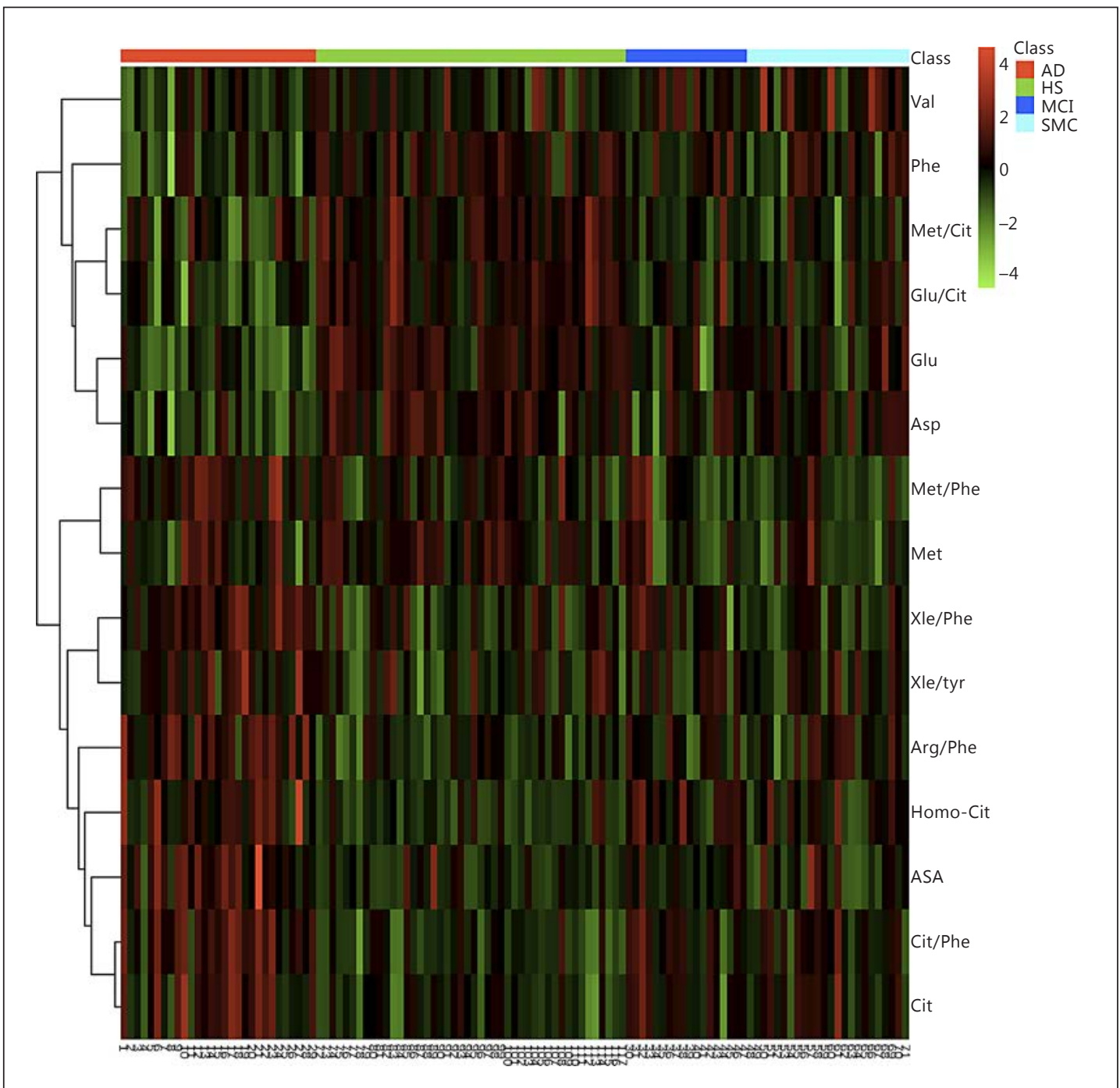

Fig. 4. Heat map of the best metabolites and molar ratios discriminating the 4 groups of subjects. The heat map obtained via multivariate analysis using the random forest algorithm shows the variations between the 4 classes of subjects in the best 15 biomarkers. AD, Alzheimer disease; MCI, mild cognitive impairment; SMC, subjective memory complaint; HS, healthy subjects. For explanations of the remaining abbreviations, see Subjects and Methods.

(AUC $=0.901)$ or the first $5(A U C=0.930)$ biomarkers (Fig. 5b). In online supplementary Figures 1 and 2, the results of the pairwise discriminant analysis using multivariate ROC curves are reported. The discriminant power using 10 or 20 biomarkers was good for the comparisons $\mathrm{AD}$ versus SMC (AUC $=0.86$ and 0.88 , respectively), $\mathrm{HS}$ versus $\mathrm{MCI}$ ( $\mathrm{AUC}=0.79$ and 0.80 , respectively), HS versus SMC (AUC $=0.75$ and 0.78 , respectively), and AD versus $\mathrm{MCI}(20$ biomarkers, $\mathrm{AUC}=0.75)$, and insufficient for $\mathrm{MCI}$ versus $\mathrm{SMC}(\mathrm{AUC}=0.51$ and 0.52 , respectively).

A ROC curve-based model evaluation (Tester) showed that the model including 10 biomarkers had a very good diagnostic power with an AUC of 0.958 in discriminating AD from HS (Fig. 6a). The cross-validation with the random forest algorithm showed an average prediction accuracy of 0.872 (Fig. $6 \mathrm{~b}$ ), and in the permutation tests $(n=1,000)$, none of the results was better than the original one ( $p<0.001$; Fig. $6 c)$. 
Fig. 5. Multivariate ROC curves of the best biomarkers (Explorer). ROC curve analysis shows that the combination of 10 biomarkers Glu, Glu/Cit, Asp, Phe, Cit/Phe, Arg/Phe, ASA, Homo-Cit, Xle/Phe, and Cit reached very good power in discriminating $\mathrm{AD}$ from $\mathrm{HS}$ (AUC $=0.948$ ) (a). Good diagnostic power was also obtained using the first 3 (AUC $=0.901$ ) or the first 5 (AUC $=0.930$ ) biomarkers (b). AD, Alzheimer disease; HS, healthy subjects; AUC, area under the curve; $\mathrm{CI}$, confidence interval. For explanations of the remaining abbreviations, see Subjects and Methods.
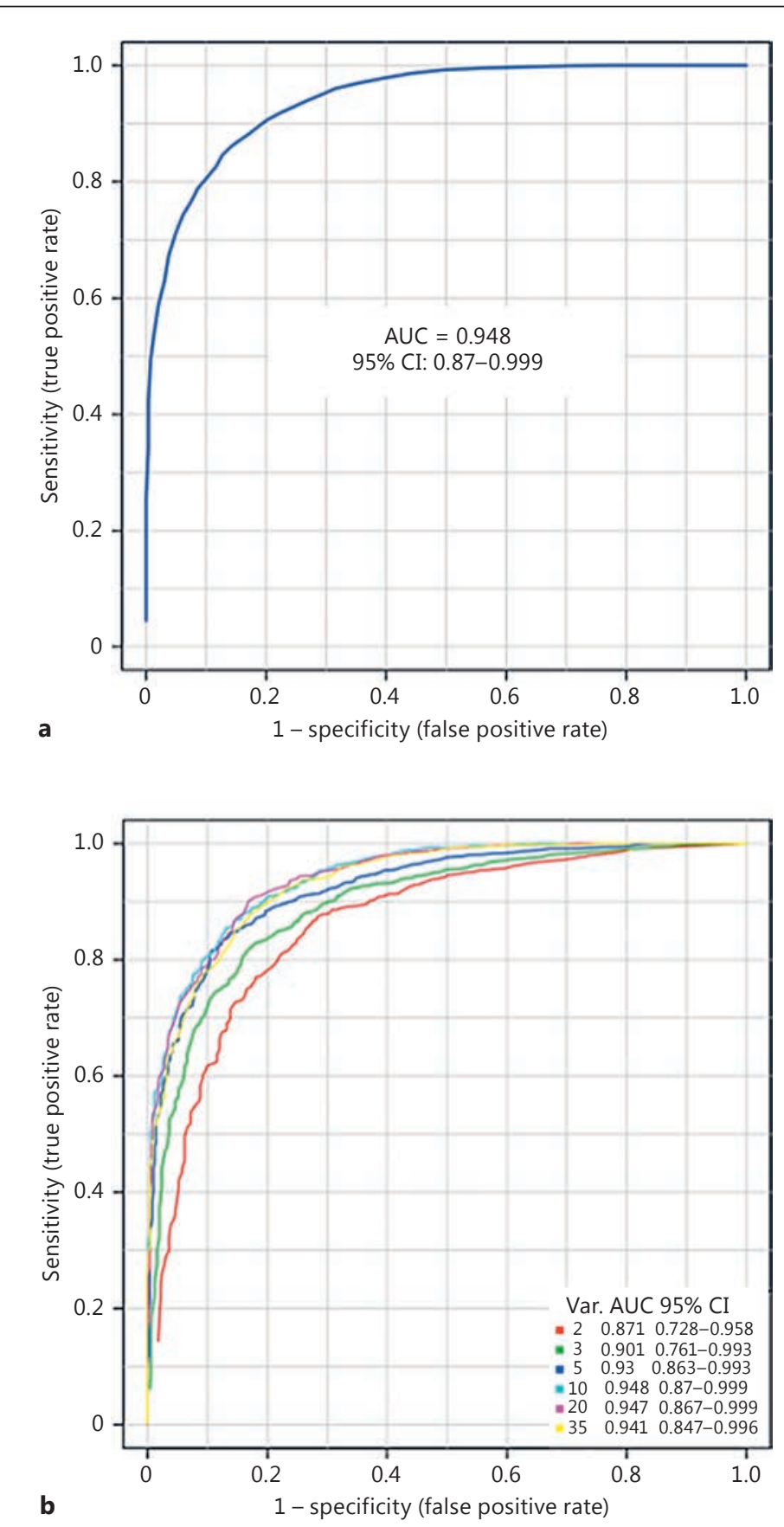

b

\section{Discussion}

The present study showed that the serum levels of several amino acids changed significantly passing from $\mathrm{HS}$ over patients at risk of $\mathrm{AD}$ (i.e., with MCI and/or SMC) to patients with $\mathrm{AD}$ along the continuum from normal aging to dementia. In particular, Glu, Asp, and Phe progressively decreased, while Cit, ASA, and Homo-Cit progressively increased from HS over SMC and MCI to AD (Table 2; Fig. 1, 2). Furthermore, several amino acids and their ratios might be used for the early detection of patients with or at risk of $\mathrm{AD}$ with a good diagnostic accuracy. 


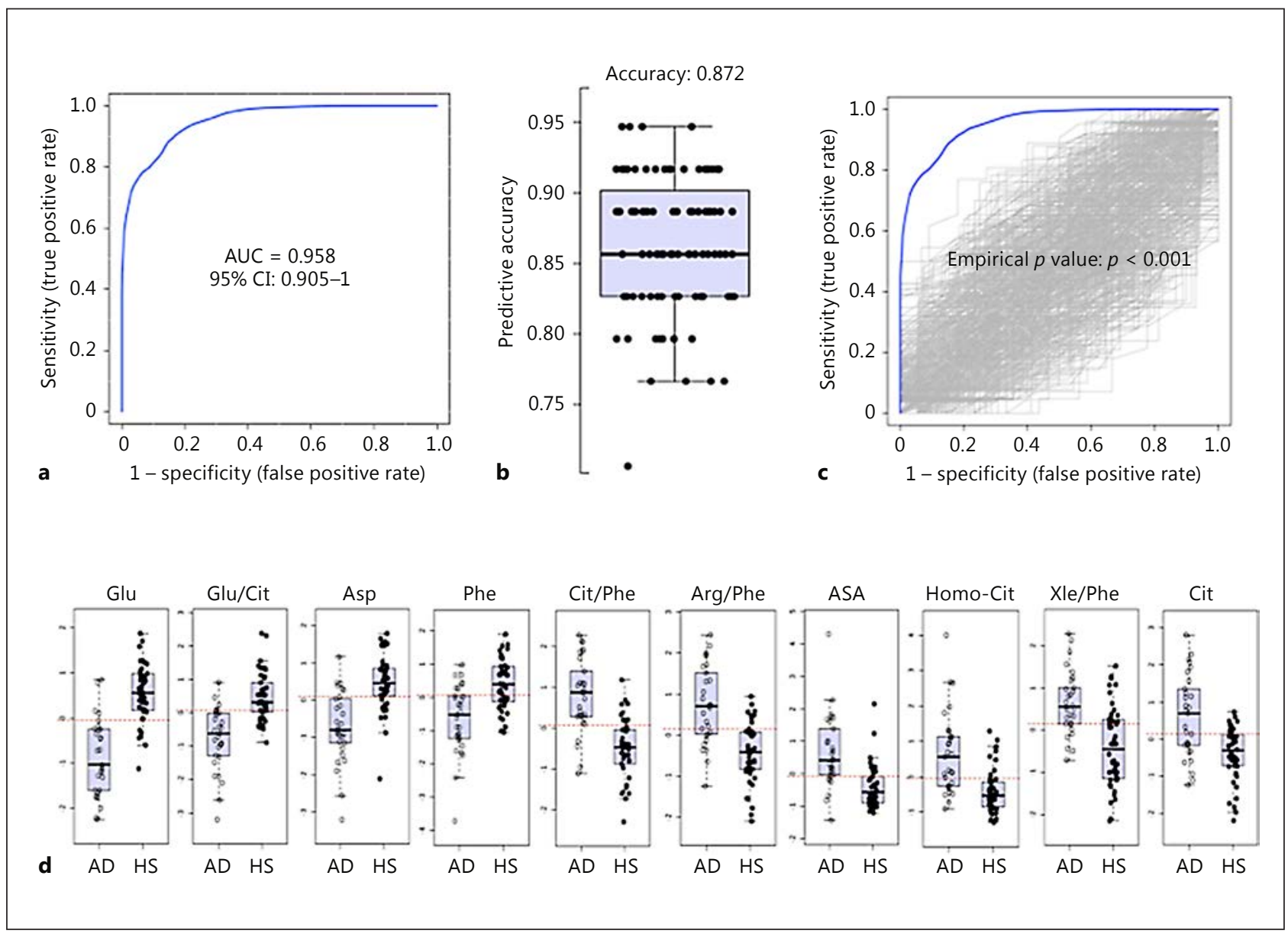

Fig. 6. Multivariate ROC curve-based model evaluation (Tester). a ROC curve analysis shows that the model including the best 10 biomarkers had a strong diagnostic power (with an AUC of 0.958) in discriminating AD from HS. b Cross-validation with the random forest algorithm showed an average prediction accuracy of 0.872. $\mathrm{c}$ In the permutation tests $(n=1,000)$, none of the results was better than the original one $(p<0.001)$. d Box plot with dots of 10 biomarkers (Glu, Asp, Phe, ASA, Homo-Cit, and Cit normalized concentrations, as well as Glu/Cit, Cit/Phe, Arg/Phe, and Xle/Phe molar ratios) included into the model. AD, Alzheimer disease; HS, healthy subjects; AUC, area under the curve; CI, confidence interval. For explanations of the remaining abbreviations, see Subjects and Methods.

In particular the multivariate model built including 6 amino acids (Glu, Asp, Phe, ASA, HomoCit, and Cit) and 4 ratios (Glu/Cit, Cit/Phe, Xle/Phe, and Arg/Phe) discriminated AD patients from HS with about $96 \%$ accuracy (Fig. 6a). These results which show that the metabolism of some physiological amino acids is impaired in AD as well as in MCI and/or SMC patients suggest the existence of a continuum in the pathophysiology of $\mathrm{AD}$ from healthy to demented.

Identifying people at risk of contracting $\mathrm{AD}$ early and as accurately as possible is a priority for the medical community. Unfortunately, current biomarkers for early disease detection such as CSF levels of $A \beta_{42}$ and total and phosphorylated tau, as well as hippocampal atrophy on MR or CT imaging and ${ }^{18} \mathrm{~F}$-fluorodeoxyglucose or amyloid PET, have a limited diagnostic accuracy [35]. Furthermore, the CSF sampling procedures are invasive and the neuroimaging techniques are expensive and time-consuming [8]. In recent years, there has been a growing interest in the detection and characterization of new biomarkers of AD using peripheral blood in order to overcome the limitations associated with the use of current biomarkers $[35,36]$. 
Peripheral blood might be a very promising substrate for biomarker searching; it is easily accessible and provides a relatively inexpensive substrate for analyses [8, 37].

A number of studies have described alterations in the metabolism of important lowmolecular-mass metabolites including amino acids [38-41] with the aim of defining the "metabolic signature" of AD [8,36]. Nevertheless, it remains unclear how the metabolic changes occurring in AD brains affect the metabolic profile of the whole body. Although several metabolites or other entities (such as proteins, transcripts, etc.) of the CNS (central nervous system) are not in an established relationship with blood, a diseased CNS might drive a peripherally detectable biosignature $[8,42]$. In serum from AD transgenic mice, GonzálezDomínguez et al. [43] recently confirmed many of alterations previously described only in brain tissue, thereby demonstrating that peripheral blood is a potential source of lowmolecular-weight biomarkers for neurodegenerative changes in the brain.

According to our results (Table 2; Fig. 1), several previous studies have reported low Glu concentrations in plasma or serum of $\mathrm{AD}[15,30,44,45]$ and $\mathrm{MCI}[15,40]$ patients in comparison with HS. In contrast, Miulli et al. [18] demonstrated a significant increase in Glu in plasma, while others did not find significant differences in serum $[17,46,47]$ of AD patients compared with that of controls. A significant Glu reduction was also measured in CSF of AD patients [20, 21, 48]; in particular, Samakashvili et al. [49] showed that the CSF Glu content progressively decreased with the severity of the disease. However, other studies found CSF Glu levels to be increased [14, 19, 50-52] or unchanged [16, 17, 46, 53]. Rupsingh et al. [54], using MR spectroscopy in vivo, found a decreased level of Glu in the hippocampus of subjects with MCI and AD compared with controls. Moreover, postmortem studies demonstrated that Glu levels were lower in hippocampus [55] and higher in frontal cortex [56] and cingulate gyrus [57] samples of AD patients compared to those of HS.

In line with our results (Table 2; Fig. 1), low concentrations of Asp have previously been reported in plasma or serum samples of $\mathrm{AD}[15,38]$ and $\mathrm{MCI}[15]$ patients, in contrast with others who found increased $[18,19]$ or unchanged $[17,21]$ plasma or serum levels of Asp in AD compared to HS. Martinez et al. [21] reported increased levels of Asp in the CSF of AD patients, in disagreement with other authors, who found low $[20,51]$ or unchanged $[16,52]$ Asp levels. Several postmortem studies [56-58] demonstrated low levels of Asp in AD patients compared to HS.

Contradictory results were also reported for CSF or blood levels of Phe, which have been found increased [59,60], reduced [38], or unaffected [21, 46, 53] in AD patients compared to HS. Ravaglia et al. [59] reported significantly high plasma levels of Phe also in MCI patients. Postmortem studies reported that Phe levels were significantly lower [61] or higher [57] in brains of AD patients compared to HS.

The serum reduction of Tyr and Val was in agreement with some reports [38], but in disagreement with others who found high [53] or unchanged concentrations $[17,21,44,46$, $50,52,59]$.

Differently from Asp, Glu, Phe, Tyr, and Val, we found that Cit, ASA, and Homo-Cit serum levels progressively increased from HS over SMC and MCI to AD; however, only the comparison HS versus AD was always significant (Table 2; Fig. 2). Previous studies also observed that in plasma [62] and CSF [46, 60], Cit occurred at significantly greater concentrations in AD patients compared to HS, while others $[17,48]$ did not find significant differences. In contrast with other authors $[15,17,59]$, we found only a trend towards increased levels of Arg in AD.

The variable and often contrasting results reported above might have several causes, including the multiplicity of methodological approaches, different numbers of observations, and the clinical features of the studied subjects. However, these contrasting results strengthen the hypothesis that $\mathrm{AD}$ is a heterogeneous and multifactorial disorder, resulting from a combination of multigenic susceptibility and environmental influences. In other words, the noso- 
logical entity of AD probably includes different pathological subtypes, each one with its own molecular mechanisms, prognosis, and therapy response.

The results of present study (in particular the decrease in Glu, Asp, Phe, Tyr, and Val, with the increase in Cit, ASA, and Homo-Cit, from HS to AD) may suggest that at least in a subgroup of $\mathrm{AD}$ patients the pathogenic mechanism is characterized by an increased use of some ketogenic and/or glucogenic amino acids for energetic purposes. A body of evidence shows that the cerebral glucose metabolism is perturbed in $\mathrm{AD}$, and in such circumstances, amino acids might be used as an energy substrate [63]. The excess of ammonium ions produced by the amino acid catabolism might saturate the urea cycle, causing an increased synthesis of Cit and ASA upstream to formation of Arg (slightly but not significantly elevated) and Orn (within normal range) (Table 2). The amount of ammonium ions that escapes the urea cycle might be transformed into Homo-Cit, thereby increasing its serum levels (Table 2). The carbon skeletons of ketogenic and/or glucogenic amino acids might be broken down into metabolites that enter the tricarboxylic acid (TCA) cycle for the production of energy. The finding that all urea cycle enzyme genes were expressed in AD brains, and that the expression of arginase 2 and ornithine transcarbamylase was higher in AD brains than in controls [64], supports our hypothesis. The observation of a possible upregulation of the TCA cycle in neurodegenerative dementia, inferred from the marginal increase in several intermediates of such a cycle in the serum of demented patients [47], also substantiates our hypothesis.

Similarly to our study, Mapstone et al. [39] identified a panel of 10 metabolites that were highly predictive of conversion to AD (AUC $=0.92$ ) in a group of cognitively normal older individuals. However, Casanova et al. [65], using the metabolite panel and the platform of Mapstone et al. [39], failed to replicate the same high prediction performance in 2 independent cohorts with a total of 193 participants (AUC max. $=0.642$ ). These findings further support the hypothesis that AD is a complex, multifactorial disorder, including different pathological subtypes. For these reasons, according to Casanova et al. [65], we suggest that biomarker panels identified in small exploratory studies, including our 10-biomarker panel discriminating AD patients from HS with about 96\% accuracy (Fig. 6a), need large-scale and well-designed validation to establish their clinical utility.

In conclusion, the results of the present study suggest that ketogenic and/or glucogenic amino acids such as Asp, Glu, Val, Phe, and Tyr might progressively decrease, while urea cycle amino acids such as Cit and ASA might progressively increase, along the continuum from cognitively normal individuals over SMC and MCI to AD patients, and that the pathogenic mechanisms might include the utilization of amino acids entering the TCA cycle for fuel sources. Our results also suggest that several amino acids and their ratios might help to identify patients before phenotype conversion to AD with a good diagnostic accuracy. However, considering the complex, multifactorial nature of $\mathrm{AD}$, further validation on greater samples in longitudinal studies is needed before application to clinical practice.

\section{Acknowledgements}

The authors are grateful to Mariella Filangieri for her valuable assistance and to Regione Molise (Italy) for funding this research (CUP H11J10000060002).

\section{Disclosure Statement}

The authors declare no conflict of interest. 


\section{References}

1 Alzheimer's Association: 2016 Alzheimer's disease facts and figures. 2016. http://www.alz.org/documents_ custom/2016-facts-and-figures.pdf (accessed November 26, 2016).

-2 Rosén C, Hansson O, Blennow K, Zetterberg H: Fluid biomarkers in Alzheimer's disease - current concepts. Mol Neurodegener 2013;8:20.

-3 Sperling R, Mormino E, Johnson K: The evolution of preclinical Alzheimer's disease: implications for prevention trials. Neuron 2014;84:608-622.

4 Susanto T-A, Pua E-P, Zhou J; Alzheimer's Disease Neuroimaging Initiative: Cognition, brain atrophy, and cerebrospinal fluid biomarkers changes from preclinical to dementia stage of Alzheimer's disease and the influence of apolipoprotein E. J Alzheimers Dis 2015;45:253-268.

5 Dubois B, Hampel H, Feldman HH, Scheltens P, Aisen P, Andrieu S, et al; Proceedings of the Meeting of the International Working Group (IWG) and the American Alzheimer's Association on "The Preclinical State of AD"; July 23, 2015; Washington DC, USA: Preclinical Alzheimer's disease: definition, natural history, and diagnostic criteria. Alzheimers Dement 2016;3:292-323.

6 Petersen RC: Mild cognitive impairment as a diagnostic entity. J Intern Med 2004;256:183-194.

7 Abdulrab K, Heun R: Subjective memory impairment. A review of its definitions indicates the need for a comprehensive set of standardised and validated criteria. Eur Psychiatry 2008;23, 321-330.

$>8$ Fiandaca MS, Mapstone ME, Cheema AK, Federoff HJ: The critical need for defining preclinical biomarkers in Alzheimer's disease. Alzheimers Dement 2014;10(suppl):S196-S212.

-9 Lista S, Molinuevo J-L, Cavedo E, Rami L, Amouyel P, Teipel S-J, Garaci F, Toschi N, Habert M-O, Blennow K, Zetterberg H, O’Bryant S, Johnson L, Galluzzi S, Bokde A-L, Broich K, Herholz K, Bakardjian H, Dubois B, Jessen F, Carrillo M-C, Aisen P-S, Hampel H: Evolving evidence for the value of neuroimaging methods and biological markers in subjects categorized with subjective cognitive decline. J Alzheimers Dis 2015;48(suppl 1):S171S191.

10 Khan T-K, Alkon D-L: Alzheimer's disease cerebrospinal fluid and neuroimaging biomarkers: diagnostic accuracy and relationship to drug efficacy. J Alzheimers Dis 2015;46:817-836.

11 Ellis B, Hye A, Snowden S-G: Metabolic modifications in human biofluids suggest the involvement of sphingolipid, antioxidant, and glutamate metabolism in Alzheimer's disease pathogenesis. J Alzheimers Dis 2015;46: 313-327.

12 Wu G: Amino acids: metabolism, functions, and nutrition. Amino Acids 2009;37:1-17.

13 Shimomura Y, Kitaura Y, Kadota Y, Ishikawa T, Kondo Y, Xu M, Ota M, Morishita Y, Bariuan J-V, Zhen H: Novel physiological functions of branched-chain amino acids. J Nutr Sci Vitaminol (Tokyo) 2015;61(suppl):S112S114.

-14 Kaiser E, Schoenknecht P, Kassner S, Hildebrandt W, Kinscherf R, Schroeder J: Cerebrospinal fluid concentrations of functionally important amino acids and metabolic compounds in patients with mild cognitive impairment and Alzheimer's disease. Neurodegener Dis 2010;7:251-259.

-15 Olazarán J, Gil-de-Gómez L, Rodríguez-Martín A, Valentí-Soler M, Frades-Payo B, Marín-Muñoz J, Antúnez C, Frank-García A, Acedo-Jiménez C, Morlán-Gracia L, Petidier-Torregrossa R, Guisasola M-C, Bermejo-Pareja F, Sánchez-Ferro Á, Pérez-Martínez D-A, Manzano-Palomo S, Farquhar R, Rábano A, Calero M: A blood-based, 7-metabolite signature for the early diagnosis of Alzheimer's disease. J Alzheimers Dis 2015;45:1157-1173.

16 Smith C-C, Bowen D-M, Francis P-T, Snowden J-S, Neary D: Putative amino acid transmitters in lumbar cerebrospinal fluid of patients with histologically verified Alzheimer's dementia. J Neurol Neurosurg Psychiatry 1985;48:469-471.

17 Fonteh AN, Harrington RJ, Tsai A, Liao P, Harrington MG: Free amino acid and dipeptide changes in the body fluids from Alzheimer's disease subjects. Amino Acids 2007;32:213-224.

18 Miulli D-E, Norwell D-Y, Schwartz F-N: Plasma concentrations of glutamate and its metabolites in patients with Alzheimer's disease. J Am Osteopath Assoc 1993;93:670-676.

-19 Jiménez-Jiménez F-J, Molina JA, Gómez P, Vargas C, de Bustos F, Benito-León J, Tallón-Barranco A, Ortí-Pareja M, Gasalla T, Arenas J: Neurotransmitter amino acids in cerebrospinal fluid of patients with Alzheimer's disease. J Neural Transm 1998;105:269-277.

20 Tohgi H, Abe T, Takahashi S, Kimura M: A selective reduction of excitatory amino acids in cerebrospinal fluid of patients with Alzheimer type dementia compared with vascular dementia of the Binswanger type. Neurosci Lett 1992;141:5-8.

-21 Martinez M, Frank A, Diez-Tejedor E, Hernanz A: Amino acid concentrations in cerebrospinal fluid and serum in Alzheimer's disease and vascular dementia. J Neural Transm Park Dis Dement Sect 1993;6:1-9.

22 McKhann G-M, Knopman D-S, Chertkow H, Hyman B-T, Jack C-R Jr, Kawas C-H, Klunk W-E, Koroshetz W-J, Manly J-J, Mayeux R, Mohs R-C, Morris J-C, Rossor M-N, Scheltens P, Carrillo M-C, Thies B, Weintraub S, Phelps $\mathrm{C}-\mathrm{H}$ : The diagnosis of dementia due to Alzheimer's disease: recommendations from the National Institute on Aging-Alzheimer's Association workgroups on diagnostic guidelines for Alzheimer's disease. Alzheimers Dement 2011;7:263-269.

23 Folstein M-F, Folstein S-E, McHugh P-R: “Mini-mental state”. A practical method for grading the cognitive state of patients for the clinician. J Psychiatr Res 1975;12:189-198.

24 Berg L: Clinical Dementia Rating (CDR). Psychopharmacol Bull 1988;24:637-639. 
Albert MS, DeKosky ST, Dickson D, Dubois B, Feldman HH, Fox NC, Gamst A, Holtzman DM, Jagust WJ, Petersen RC, Snyder PJ, Carrillo MC, Thies B, Phelps CH: The diagnosis of mild cognitive impairment due to Alzheimer's disease: recommendations from the National Institute on Aging-Alzheimer's Association workgroups on diagnostic guidelines for Alzheimer's disease. Alzheimers Dement 2011;7:270-279.

26 Rey A: L'examen clinique en psychologie: Memorisation d'une serie de 15 mots en 5 repetitions. Paris, Presses Universitaires de France, 1958.

-27 Spinnler H, Tognoni G: Standardizzazione e taratura italiana di test neuropsicologici. Ital J Neurol Sci 1987;suppl 8:1-120.

28 Crook T-H 3rd, Feher E-P, Larrabee G-J: Assessment of memory complaint in age-associated memory impairment: the MAC-Q. Int Psychogeriatr 1992;4:165-176.

29 Almeida O-P, Almeida S-A: Short versions of the Geriatric Depression Scale: a study of their validity for the diagnosis of a major depressive episode according to ICD-10 and DSM-IV. Int J Geriatr Psychiatry 1999;14: 858-865.

30 la Marca G, Malvagia S, Toni S, Piccini B, Di Ciommo V, Bottazzo G-F: Children who develop type 1 diabetes early in life show low levels of carnitine and amino acids at birth: does this finding shed light on the etiopathogenesis of the disease? Nutr Diabetes 2013;3:e94.

-31 Dietzen D-J, Rinaldo P, Whitley R-J, Rhead W-J, Hannon W-H, Garg U-C, Lo S-F, Bennett M-J: National academy of clinical biochemistry laboratory medicine practice guidelines: follow-up testing for metabolic disease identified by expanded newborn screening using tandem mass spectrometry; executive summary. Clin Chem 2009;55:1615-1626.

-32 Xia J, Sinelnikov I-V, Han B, and Wishart D-S: MetaboAnalyst 3.0 - making metabolomics more meaningful. Nucleic Acids Res 2015;43(W1):W251-W257.

33 Xia J, Broadhurst D-I, Wilson M, Wishart D-S: Translational biomarker discovery in clinical metabolomics: an introductory tutorial. Metabolomics 2013;9:280-299.

34 Jones C-M, Athanasiou T: Summary receiver operating characteristic curve analysis techniques in the evaluation of diagnostic tests. Ann Thorac Surg 2005;79:16-20.

-35 Scheltens P, Blennow K, Breteler M-M, de Strooper B, Frisoni G-B, Salloway S, Van der Flier W-M: Alzheimer's disease. Lancet 2016;388:505-517.

-36 Davinelli S, Intrieri M, Russo C, Di Costanzo A, Zella D, Bosco P, Scapagnini G: The “Alzheimer's disease signature": potential perspectives for novel biomarkers. Immun Ageing 2011;8:7.

37 Henriksen K, O’Bryant S-E, Hampel H, Trojanowski J-Q, Montine T-J, Jeromin A, Blennow K, Lönneborg A, Wyss-Coray T, Soares H, Bazenet C, Sjögren M, Hu W, Lovestone S, Karsdal M-A, Weiner M-W; Blood-Based Biomarker Interest Group: The future of blood-based biomarkers for Alzheimer's disease. Alzheimers Dement 2014;10:115-131.

-38 González-Domínguez R, García-Barrera T, Gómez-Ariza J-L: Metabolite profiling for the identification of altered metabolic pathways in Alzheimer's disease. J Pharm Biomed Anal 2015;107:75-81.

-39 Mapstone M, Cheema A-K, Fiandaca M-S, Zhong X, Mhyre T-R, MacArthur L-H, Hall W-J, Fisher S-G, Peterson D-R, Haley J-M, Nazar M-D, Rich S-A, Berlau D-J, Peltz C-B, Tan M-T, Kawas C-H, Federoff H-J: Plasma phospholipids identify antecedent memory impairment in older adults. Nat Med 2014;20:415-418.

-40 Wang G, Zhou Y, Huang F-J, Tang H-D, Xu X-H, Liu J-J, Wang Y, Deng Y-L, Ren R-J, Xu W, Ma J-F, Zhang Y-N, Zhao A-H, Chen S-D, Jia W: Plasma metabolite profiles of Alzheimer's disease and mild cognitive impairment. J Proteome Res 2014;13:2649-2658.

-41 Trushina E, Dutta T, Persson X-M, Mielke M-M, Petersen R-C: Identification of altered metabolic pathways in plasma and CSF in mild cognitive impairment and Alzheimer's disease using metabolomics. PLoS One 2013;8:e63644.

42 Tylee D-S, Kawaguchi D-M, Glatt S-J: On the outside, looking in: a review and evaluation of the comparability of blood and brain “-omes.” Am J Med Genet B Neuropsychiatr Genet 2013;162B: 595-603.

-43 González-Domínguez R, García-Barrera T, Vitorica J, Gómez-Ariza J-L: Application of metabolomics based on direct mass spectrometry analysis for the elucidation of altered metabolic pathways in serum from the APP/ PS1 transgenic model of Alzheimer's disease. J Pharm Biomed Anal 2015;107:378-385.

-44 Basun H, Forssell L-G, Almkvist O, Cowburn R-F, Eklöf R, Winblad B, Wetterberg L: Amino acid concentrations in cerebrospinal fluid and plasma in Alzheimer's disease and healthy control subjects. J Neural Transm Park Dis Dement Sect 1990;2:295-304

-45 González-Domínguez R, García-Barrera T, Gómez-Ariza J-L: Using direct infusion mass spectrometry for serum metabolomics in Alzheimer's disease. Anal Bioanal Chem 2014;406:7137-7148.

46 Mochizuki Y, Oishi M, Hara M, Takasu T: Amino acid concentration in dementia of the Alzheimer type and multi-infarct dementia. Ann Clin Lab Sci 1996;26:275-278.

47 Tsuruoka M, Hara J, Hirayama A, Sugimoto M, Soga T, Shankle W-R, Tomita M: Capillary electrophoresis-mass spectrometry-based metabolome analysis of serum and saliva from neurodegenerative dementia patients. Electrophoresis 2013;34:2865-2872.

48 Kuiper M-A, Teerlink T, Visser J-J, Bergmans P-L, Scheltens P, Wolters E-C: L-Glutamate, L-arginine and L-citrulline levels in cerebrospinal fluid of Parkinson's disease, multiple system atrophy, and Alzheimer's disease patients. J Neural Transm 2000;107:183-189. 
49 Samakashvili S, Ibáñez C, Simó C, Gil-Bea F-J, Winblad B, Cedazo-Mínguez A, Cifuentes A: Analysis of chiral amino acids in cerebrospinal fluid samples linked to different stages of Alzheimer disease. Electrophoresis 2011;32:2757-2764.

50 Fisher G, Lorenzo N, Abe H, Fujita E, Frey W-H, Emory C, Di Fiore MM, D’Aniello A: Free D- and L-amino acids in ventricular cerebrospinal fluid from Alzheimer and normal subjects. Amino Acids 1998;15:263-269.

-51 D’Aniello A, Fisher G, Migliaccio N, Cammisa G, D’Aniello E, Spinelli P: Amino acids and transaminases activity in ventricular CSF and in brain of normal and Alzheimer patients. Neurosci Lett 2005;388:49-53.

52 Pomara N, Singh R, Deptula D, Chou J-C, Schwartz M-B, LeWitt P-A: Glutamate and other CSF amino acids in Alzheimer's disease. Am J Psychiatry 1992;149:251-254.

53 Degrell I, Hellsing K, Nagy E, Niklasson F: Amino acid concentrations in cerebrospinal fluid in presenile and senile dementia of Alzheimer type and multi-infarct dementia. Arch Gerontol Geriatr 1989;9:123-135.

54 Rupsingh R, Borrie M, Smith M, Wells J-L, Bartha R: Reduced hippocampal glutamate in Alzheimer disease. Neurobiol Aging 2011;32:802-810.

55 Liu P, Fleete M-S, Jing Y, Collie N-D, Curtis M-A, Waldvogel H-J, Faull R-L, Abraham W-C, Zhang H: Altered arginine metabolism in Alzheimer's disease brains. Neurobiol Aging 2014;35:1992-2003.

-56 Paglia G, Stocchero M, Cacciatore S, Lai S, Angel P, Alam M-T, Keller M, Ralser M, Astarita G: Unbiased metabolomic investigation of Alzheimer's disease brain points to dysregulation of mitochondrial aspartate metabolism. J Proteome Res 2016;15:608-618.

57 Xu J, Begley P, Church S-J, Patassini S, Hollywood K-A, Jüllig M, Curtis M-A, Waldvogel H-J, Faull R-L, Unwin R-D, Cooper G-J: Graded perturbations of metabolism in multiple regions of human brain in Alzheimer's disease: snapshot of a pervasive metabolic disorder. Biochim Biophys Acta 2016;1862:1084-1092.

-58 D’Aniello A, Lee J-M, Petrucelli L, Di Fiore M-M: Regional decreases of free D-aspartate levels in Alzheimer's disease. Neurosci Lett 1998;250:131-134.

59 Ravaglia G, Forti P, Maioli F, Bianchi G, Martelli M, Talerico T, Servadei L, Zoli M, Mariani E: Plasma amino acid concentrations in patients with amnestic mild cognitive impairment or Alzheimer disease. Am J Clin Nutr 2004;80:483-488.

60 Czech C, Berndt P, Busch K, Schmitz O, Wiemer J, Most V, Hampel H, Kastler J, Senn H: Metabolite profiling of Alzheimer's disease cerebrospinal fluid. PLoS One 2012;7:e31501.

61 Takayama T, Mochizuki T, Todoroki K, Min J-Z, Mizuno H, Inoue K, Akatsu H, Noge I, Toyo'oka T: A novel approach for LC-MS/MS-based chiral metabolomics fingerprinting and chiral metabolomics extraction using a pair of enantiomers of chiral derivatization reagents. Anal Chim Acta 2015;898:73-84.

-62 Molina J-A, Jiménez-Jiménez F-J, Vargas C, Gómez P, de Bustos F, Ortí-Pareja M, Tallón-Barranco A, BenitoLeón J, Arenas J, Enríquez-de-Salamanca R: Cerebrospinal fluid levels of non-neurotransmitter amino acids in patients with Alzheimer's disease. J Neural Transm 1998;105:279-286.

63 Chen Z, Zhong C: Decoding Alzheimer's disease from perturbed cerebral glucose metabolism: implications for diagnostic and therapeutic strategies. Prog Neurobiol 2013;108:21-43.

64 Hansmannel F, Sillaire A, Kamboh M-I, Lendon C, Pasquier F, Hannequin D, Laumet G, Mounier A, Ayral A-M, DeKosky S-T, Hauw J-J, Berr C, Mann D, Amouyel P, Campion D, Lambert J-C: Is the urea cycle involved in Alzheimer's disease? J Alzheimers Dis 2010;21:1013-1021.

-65 Casanova R, Varma S, Simpson B, Kim M, An Y, Saldana S, Riveros C, Moscato P, Griswold M, Sonntag D, Wahrheit J, Klavins K, Jonsson P-V, Eiriksdottir G, Aspelund T, Launer LJ, Gudnason V, Legido Quigley C, Thambisetty M: Blood metabolite markers of preclinical Alzheimer's disease in two longitudinally followed cohorts of older individuals. Alzheimers Dement 2016;12:815-822. 